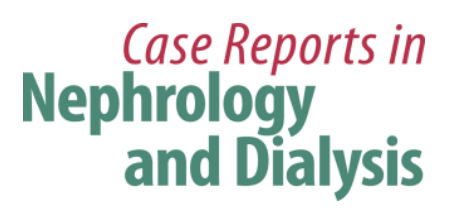

Case Rep Nephrol Dial 2016;6:83-88

DOI: $10.1159 / 000446393$

Publisnea onine: June 10, 2016

(c) 2016 The Author(s)
Published by S. Karger AG, Basel
www.karger.com/cnd

Karger
Open access

This article is licensed under the Creative Commons Attribution-NonCommercial 4.0 International License (CC BY-NC) (http://www.karger.com/Services/OpenAccessLicense). Usage and distribution for commercial purposes requires written permission.

\title{
Classical Presentation of Acute Pyelonephritis in a Case of Brucellosis
}

\author{
Wadha Alfouzan ${ }^{a, b}$ Sara Al-Sahali ${ }^{a}$ Hawra'a Sultan ${ }^{c}$ Rita Dhar ${ }^{a}$ \\ ${ }^{a}$ Microbiology Unit, Department of Laboratories, Al-Farwania Hospital, Kuwait \\ City, Kuwait; ${ }^{b}$ Department of Microbiology, Faculty of Medicine, Kuwait University, \\ Kuwait City, Kuwait; ${ }^{\mathrm{C} D e p a r t m e n t}$ of Medicine, Al-Farwania Hospital, Kuwait City, Kuwait
}

\section{Keywords}

Acute pyelonephritis · Brucellosis · Brucella spp. · Bacteremia

\begin{abstract}
Although Brucella species is known to affect almost all organs in humans, renal involvement presenting as acute pyelonephritis remains a rare entity in brucellosis. We report the case of a female patient who presented with symptoms of fever with chills, right loin pain and dysuria in the emergency room. Blood cultures drawn at the time of admission grew Brucella spp., but no organisms were isolated from urine culture although urinalysis data was indicative of urinary tract infection. Empiric therapy with piperacillin/tazobactam plus gentamicin relieved her symptoms. However, the treatment was switched to doxycycline plus rifampicin once the blood culture result was obtained.

(C) 2016 The Author(s)

Published by S. Karger AG, Basel
\end{abstract}

\section{Introduction}

Brucellosis is one of the most common anthropozoonotic diseases reported globally [1]. The infection is transmitted to humans by exposure to infected materials, especially vaginal secretions, placenta waste, fetus, etc. from animals, such as sheep, cattle, goats, pigs and others. Also, the infection can be acquired by consumption of unpasteurized milk and milk products derived from infected animals as well as laboratory-related accidents [2, 3]. Brucel- 


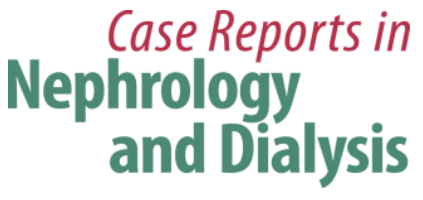

Case Rep Nephrol Dial 2016;6:83-88 $10.1159 / 000446393$

Alfouzan et a : Classical Presentation of Acute Pyeloneph

Alfouzan et al.: Classical Presentation of Acute Pyelonephritis in a Case of Brucellosis

losis can cause high morbidity as much in humans as in farm animals leading to great economic losses. Brucella spp. is classified as a Class B Bioterrorist agent, posing a major public health problem in many developing and developed countries [4].

Brucellosis is a febrile systemic illness with protean manifestations and forms an important component of list of conditions listed under 'fever of unknown origin'. The Clinical presentation can vary from asymptomatic infection to fever, night sweats, body aches and rarely lead to complications associated with the osteoarticular, hematological, hepatobiliary, gastrointestinal and genitourinary system [5]. Involvement of cardiac, ocular, skin and mucous membranes or central nervous system has also been increasingly reported from endemic countries [4]. Although osteoarticular involvement remains to be the most frequent complication of brucellosis, genitourinary complications are usually found on the other end of the spectrum $[5,6]$. While there are several reports on epididymo-orchitis among genitourinary complications of brucellosis in the literature, there is dearth of articles on pyelonephritis. To the best of our knowledge, this is the first report of pyelonephritis due to Brucella spp. from Kuwait.

There are currently six classical species within the genus Brucella, namely B. abortus, $B$. melitensis, $B$. ovis, B. canis, B. suis and B. neotome. The laboratory diagnosis of brucellosis is usually by isolation of the bacteria from the clinical sample or detection of anti-brucella antibodies in the serum of the patient. However, newer methods are being employed for rapid identification and speciation of Brucella [7, 8].

\section{Case Report}

A 38-year-old female, Kuwaiti patient presented with symptoms of fever with rigors, right loin pain and dysuria with change in urine color and foul odor of 5 days' duration. She had visited a private clinic 3 weeks earlier for persistent fever and received parenteral antibiotics. Although she responded initially with improvement in her condition, she had a relapse of her symptoms and presented to the emergency room of our hospital. On physical examination, the patient was found to be febrile with oral temperature $38.5^{\circ} \mathrm{C}$, heart rate 93 bpm, and blood pressure 117/65 mm Hg. Chest and cardiac auscultation did not reveal any abnormality. On palpation, the abdomen was found to be soft and lax but there was right renal angle tenderness. Laboratory investigations revealed WBC $4.9 \times 10^{9} / 1$ (neutrophils 45.8\%; lymphocytes 51.0\%), hemoglobin $121 \mathrm{~g} / \mathrm{l}$, platelets $160 \times 10^{9} / \mathrm{l}$, urea $2.7 \mathrm{mmol} / \mathrm{l}$, creatinine $54 \mu \mathrm{mol} / \mathrm{l}$, sodium $135 \mathrm{mmol} / \mathrm{l}$, potassium $4 \mathrm{mmol} / \mathrm{l}$ and C-reactive protein 95 $\mathrm{mg} / \mathrm{l}$ (normal range, $0-8 \mathrm{mg} / \mathrm{l}$ ). Of two sets of blood samples collected in aerobic and anaerobic bottles (BACTEC Plus Aerobic/F, BACTEC Plus Anaerobic/F), only one of the aerobic bottles was flagged positive in the blood culture instrument (BD BACTEC 9240) after $>48 \mathrm{~h}$ of incubation. The Gram-stained smear of the sample from the positive blood culture bottle showed presence of Gram-negative coccobacilli in clumps, and culture on blood and chocolate agar plates also grew the same organism. The isolate was positive for catalase, oxidase and urease tests and also agglutinated with positive control serum from Brucelloslide test (bioMerieux, France). The preliminary identification of the isolate was Brucella spp., which was confirmed by PCR in the reference laboratory in Kuwait. Urinalysis revealed leukocytes $500 / \mu \mathrm{l}$ (normal $<10 / \mu \mathrm{l}$ ), erythrocytes $250 / \mu \mathrm{l}$ (normal $<0-5 / \mu \mathrm{l}$ ) and protein $0.25 \mathrm{~g} / \mathrm{l}$ (normal $<0.1 \mathrm{~g} / \mathrm{l}$ ), while glucose and nitrate were negative. Culture of a urine sample collected at the time of admission did not yield growth of any organisms on blood and McConkey agar plates after $24 \mathrm{~h}$ of incubation. The serum sample showed anti-brucella antibodies at a titer of 
1:1,280 (Plasmatec, Dorset, UK). The patient is a known case of rheumatoid arthritis for the past 2 years, being treated with methotextrate. She also has history of recurrent urinary tract infections, which prompted her to undergo ultrasound of the abdomen in 2010, revealing a duplex right kidney and presence of calculi in the left kidney (fig. 1) and gravel in the right kidney on different occasions in the past. Ultrasound done during the present admission revealed few left renal gravels (image not added as it is not relevant to the present case scenario). In addition, she has been complaining of symptoms associated with vaginitis and seasonal bronchial asthma. From the family history it was discovered that the patient's husband often consumes unpasteurized milk from livestock, mostly sheep, which he owns on his farm. However, our patient denied having visited the farm or drinking unpasteurized milk but revealed that her husband had been complaining of fever for the past 6 weeks for which he had visited the peripheral clinic on a number of occasions and received several antibiotics with no relief. Consequently, he was summoned to our hospital for investigations and tested positive for anti-brucella antibodies (titer $=1: 320$ ).

After a clinical diagnosis of acute pyelonephritis was made in our patient in the emergency room, empirical therapy with piperacillin/tazobactam plus gentamicin was initiated. Although she did show some response initially, there was a spike of fever after $48 \mathrm{~h}$, prompting change of treatment to doxycycline plus rifampicin on the basis of a positive blood culture report.

\section{Discussion}

More often than not brucellosis follows an indolent course and since it can involve almost every system in the body it causes a significant diagnostic dilemma as it masquerades many infectious and non-infectious conditions [9]. Although brucellosis is now being rarely reported from the industrialized countries, it continues to be endemic in certain parts of the world, especially Latin America, the Mediterranean, Mid-Eastern and some Asian countries $[4,10]$. In Kuwait, brucellosis remains an endemic disease with a prevalence reaching up to 70 cases per 100,000 population [11].

To date, several articles on brucellosis in Kuwait have been published, which include varied complications involving different organs. In one of the studies, skeletal (26\%), and genital (8.5\%) changes and neurobrucellosis (7\%) were observed in the resident population in Kuwait [12]. Furthermore, during the past two decades, sporadic cases of endocarditis, myocarditis, interstitial pneumonitis, skin and soft tissue infections, thyroiditis, pancreatitis and peritonitis have also been reported from Kuwait. On similar lines, a recent report from Turkey describes different clinical presentations in human brucellosis. In their study, the authors observed varied complications, which included the hematological (58\%), osteoarticular (48\%), hepatobiliary (27\%), gastrointestinal $(10 \%)$, and genitourinary system (5\%) [6].

Among reports describing genitourinary complications, the rates vary from $2-40 \%$, and the most frequent affected site has been found to be testes and epididymus in male patients [13]. In females, pyelonephritis (86.8\%) was determined to be significantly higher as compared to males (3.1\%) in a recent large series involving 390 patients (352 male; 38 female) with genitourinary brucellosis [13]. However, the authors concluded that when the brucella cases with pyelonephritis were taken as a whole, one fourth of the cases were male patients. Hence, pyelonephritis related to brucellosis may not be considered as unique to female patients. They also reiterate the rarity of occurrence of this complication of glomerulonephritis 
in brucellosis except in patients who develop endocarditis caused by Brucella spp., where nephritis results as a complication of endocarditis [13]. Our patient did not have signs or symptoms of involvement of any other site. In the same report, dysuria, hematuria and costovertebral angle tenderness was observed in $23.8,6.7$, and $3.3 \%$ of cases, respectively. However, these included all patients (male and female) with clinical symptoms and signs of genitourinary system brucellosis. Among female patients, dysuria was observed in 55.3\% (21/38), hematuria in $26.3 \%$ and genital discharge in $10.5 \%$. The urinalysis report in our patient corroborated with signs of UTI. While there is paucity of information on urinalysis data, urine culture was found positive for Brucella spp. in 1.5\% of cases with genitourinary brucellosis [13]. Although the authors mention the media used for urine culture on which brucella was isolated, there is no reference to the duration of incubation, which is an important determinant of isolation procedure for Brucella species from clinical samples [14]. Since we were not expecting brucellosis in the present case, the blood and McConkey plates inoculated with the urine sample were discarded after $24 \mathrm{~h}$ of incubation when no microorganism was isolated.

Comorbidities, such as type 2 diabetes mellitus (19\%), HBsAg positivity (12\%), steroid therapy (4\%), and HIV (3\%) in patients with brucellosis have been reported in earlier studies $[4,14]$. However, there is not enough data to significantly associate these infections with brucellosis. Although our patient is not diabetic, she is receiving methotrexate as a therapy for rheumatoid arthritis and in addition suffers from a duplex kidney with tendency towards forming urinary stones bilaterally causing repeated urinary tract infections. Use of methotrexate, which can cause immunosuppression, could have played a pivotal role in acquisition of infection since there is experimental evidence showing that induced immunosuppression does modulate the severity and outcome of brucellosis.

While brucellosis is considered a zoonotic disease, there is a strong possibility that there is sexual dissemination among humans and we speculate that the present case might have acquired the infection from her husband since the patient vehemently denied having visited the farm in the past 6 months or having consumed unpasteurized milk unlike her spouse [15]. Since the husband did not have any localizing symptoms, he was not investigated for genital infection, such as epididymitis or orchitis. In previous studies from Turkey and Iran, family history was positive in $9.6-54 \%$ of the patients [6].

Diagnosis of genitourinary brucellosis requires a detailed history, thorough physical examination and meticulous laboratory and radiological evaluation. Pyelonephritis due to $\mathrm{Bru}$ cella spp. should be suspected in patients with signs of upper urinary tract infection who are febrile with absence of leukocytosis and with mild to moderate increase in inflammatory markers [13]. This is essential for appropriate and adequate therapy for brucellosis to prevent relapses and serious complications. During her earlier visit to a private clinic we assume that she was treated with ceftriaxone or ciprofloxacin, both of which have been shown to be effective against Brucella spp. in vitro but do not constitute specific therapy for brucellosis.

\section{Conclusion}

Given the high proportion of cases presenting with fever and complexity of the clinical manifestations of the infection, brucellosis should be considered as a differential diagnosis for fevers of unknown origin. Improved diagnostic methods could help reduce the diagnostic delay and facilitate prompt and appropriate treatment before the complications set in. 


\section{Statement of Ethics}

Consent for publication taken from the patient. All data exposing patient identification has been omitted.

\section{Disclosure Statement}

The authors have no conflicts of interest to disclose.

\section{References}

-1 Dean AS, Crump L, Greter H, Hattendorf J, Schelling E, Zinsstag J: Clinical manifestations of human brucellosis: a systematic review and meta-analysis. PLoS Negl Trop Dis 2012;69:1-9.

-2 Shimal SB, Dukhan L, Belmaker S, SibirskyD, Barrett C, Greenberg D: Human brucellosis outbreak acquired through camel milk ingestion in Southern Israel. Isr Med Assoc J 2012;14:475-478.

-3 Traxler RM, Lehman MW, Bosserman EA, Guerra MA, Smith TL: A literature review of laboratoryacquired brucellosis. J Clin Microbiol 2013;51:3055-3062.

4 Sathyanarayanan V, Razak A, Savaru K, Ananthakrishnan SB, Mukhyprana PM, Vandana KE: Clinical profile of brucellosis from a tertiary care center in Southern India. Asian Pac J Trop Med 2011;397-400 Guler S, Kokoglu OF, Ucmak H, Gul M, Ozden S, Ozkan F: Human brucellosis in Turkey: different clinical presentations. J Infect Dev Ctries 2014;8:581-588.

-6 Buzgan T, Karahocagil MK, Irmak H, Baran AI, Karsen H, Evirgen O, Akdeniz H: Clinical manifestations and complications in 1,028 cases of brucellosis: a retrospective evaluation and review of the literature. Int J Infect Dis 2010;14:469-478.

7 Mohamed ZJ, Bee Yong T, Hashim R, Mohamed NA, Hamzah SH, Ahmad N: Identification of Brucella spp. isolated from human brucellosis in Malaysia using high-resolution melt (HRM) analysis. Diagn Microbiol Infect Dis 2015;81:227-233.

-8 Al-Nakkas A, Mustafa AS, Wright SG: Large scale evaluation of a single-tube nested PCR for the laboratory diagnosis of human brucellosis in Kuwait. J Med Microbiol 2005;54(Pt 8):727-730. Franco MP, Mulder M, Gilman RH, Smits HL: Human brucellosis. Lancet Infect Dis 2007;7:775-786. Pappas G, Akritidis N, Bosilkovski M, Tsianos E: Brucellosis. N Engl J Med 2005;352:2325-2336.

10 Khateeb MI, Araj GF, Majeed SA, Lulu AR: Brucella arthritis: a study of 96 cases in Kuwait. Ann Rheum Dis 1990;49:994-998.

12 Lulu AR, Araj GF, Khateeb MI, Mustafa MY, Yusuf AR, Fenech FF: Human brucellosis in Kuwait: a prospective study of 400 cases. Q J Med 1988;66:39-54.

13 Erdem H, Elaldi N, Ak O, Gulsun S, Tekin R, Ulug M, Duygu F, Sunnrtcioglu M, Tulek N, Guler S, Cag Y, Kaya S, et al: Genitourinary brucellosis: results of a multicentric study. Clin Microbiol Infect 2014;20:0847-0853.

14 Dhar R, Dhar PM, Gafoor M: Recurrent epidermal cyst infection caused by Brucella melitensis in a diabetic patient. J Clin Microbiol 1988;26:1040-1041.

15 Meltzer E, Sidi Y, Smolen G, Banai M, Bardenstein S, Schwartz E: Sexually transmitted brucellosis in humans. Clin Infect Dis 2010;51:e12-e15. 


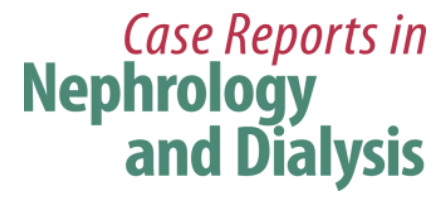

Case Rep Nephrol Dial 2016;6:83-88

(c) 2016 The Author(s). Published by S. Karger AG, Basel www.karger.com/cnd

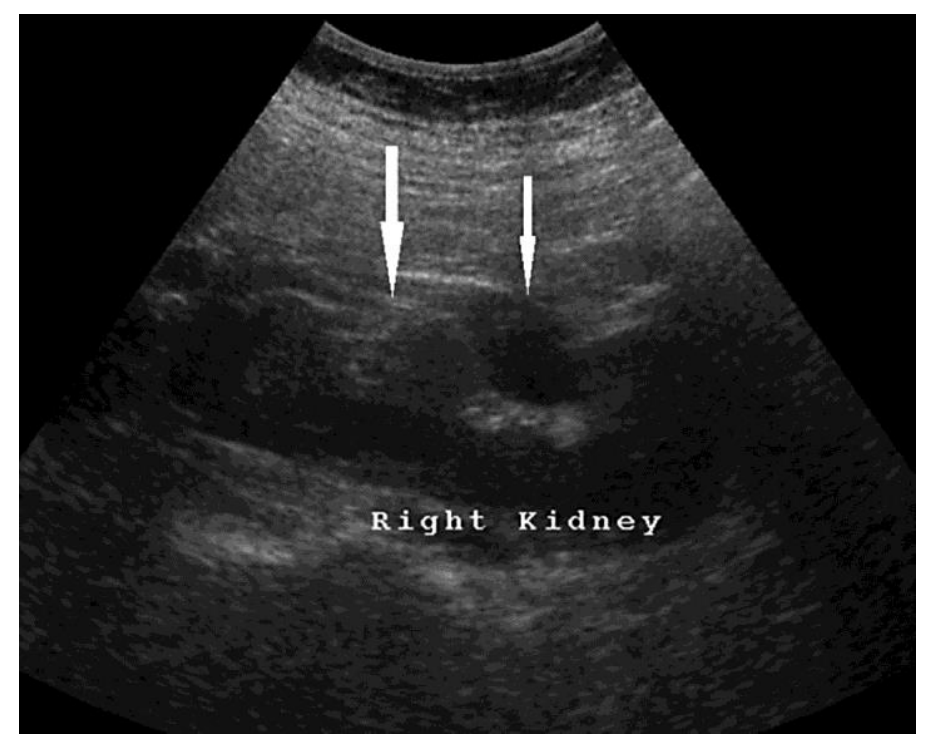

Fig. 1. Right kidney showing double moiety manifested as two partially separated collecting systems (arrows). 